\title{
Slow light fiber systems in microwave photonics
}

\author{
Luc Thévenaz ${ }^{\text {**, Sang-Hoon Chin }}{ }^{\mathrm{a}}$, \\ Perrine Berger $^{\mathrm{b}}$, Jérôme Bourderionnet ${ }^{\mathrm{b}}$, Salvador Sales ${ }^{\mathrm{c}}$, Juan Sancho-Dura ${ }^{\mathrm{c}}$ \\ ${ }^{a}$ Ecole Polytechnique Fédérale Lausanne, STI-GR-SCI-LT Station 11, 1015 Lausanne, Switzerland; \\ ${ }^{\mathrm{b}}$ Thales Research \& Technology, 1 av. Augustin Fresnel, 91767 Palaiseau Cedex, France \\ c iTEAM Institute, Universidad Politécnica de Valencia, 46022 Valencia, Spain
}

\begin{abstract}
Slow light systems are particularly attractive for analog signal processing, since their inherent limitation to a delaybandwidth product of 1 is less critical for analog systems such as those used in microwave photonics. We present here the implementation of two basic functions - phase shifting and true time delaying - fully optically controlled using stimulated Brillouin scattering in optical fibers. The combination of these two functions makes possible the implementation of true time delays without limitation on the microwave carrier frequency using the separate carrier tuning technique. This is illustrated by the implementation of the delaying system for the realization of a microwave tunable notch filter.
\end{abstract}

Keywords: Fiber optics, nonlinear fiber optics, microwave photonics, slow and fast light, stimulated Brillouin scattering.

\section{INTRODUCTION}

The realization of functionalities on microwave signals in the optical domain is an application field of photonics showing a recent growing interest regarding its substantial added value in terms of performance ${ }^{1}$. The basic concept is to modulate an optical wave by a microwave signal, then to perform a transformation of the signal in the optical domain and to eventually convert the optical signal into the electrical domain to retrieve the transformed microwave signal. This generic concept is sketched in Figure 1.

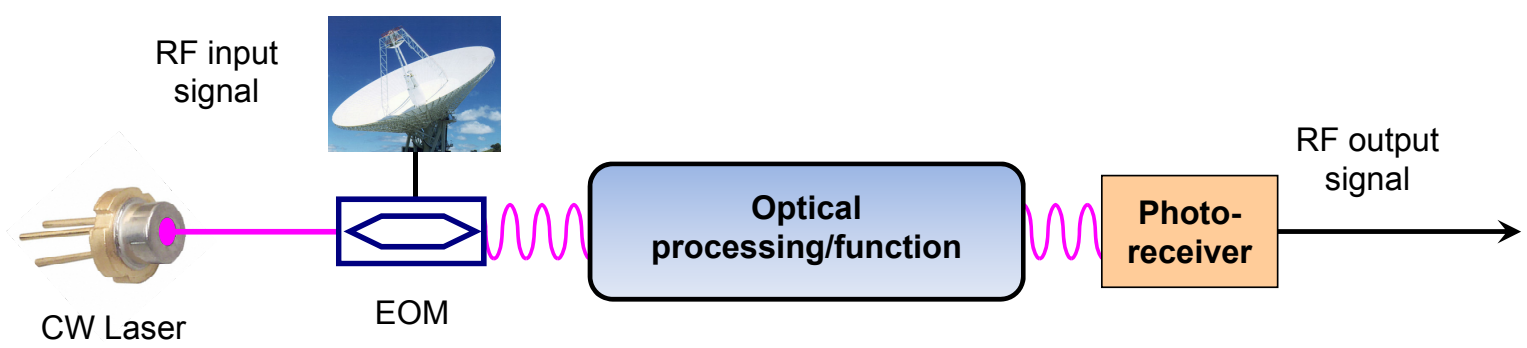

Figure 1. Concept of a microwave photonics system: the microwave signal is converted into the optical domain through an optical modulation, is then transformed using an optical processing/function to eventually return into the electrical domain through a broadband photodetection.

In full generality the spectral content of the microwave signal is made of a carrier and 2 modulation sidebands, as depicted in Figure 2 left. In the optical domain, after modulation, the full microwave signal (carrier + sidebands) will be transferred into the modulation spectral container making the optical signal sidebands. This way the optical carrier and the optical sidebands will be separated by a spectral distance corresponding to the microwave carrier frequency, and each optical sideband will contain the entire microwave signal and will spread over the bandwidth of the microwave, as shown in Figure 2 on the right. Each optical sideband contains the entire information carried by the microwave signal, so that one of the sidebands can be suppressed without loss of information. This is generally performed using a singlesideband modulation scheme or by simple optical filtering for a sufficiently high microwave carrier frequency.

*Luc.Thevenaz@EPFL:CH; phone +41 21693 4774; http://people.epfl.ch/luc.thevenaz

\footnotetext{
Advances in Slow and Fast Light IV, edited by Selim M. Shahriar, Philip R. Hemmer, Proc. of SPIE Vol. 7949, 79490B - (C) 2011 SPIE · CCC code: 0277-786X/11/\$18 doi: $10.1117 / 12.881405$
} 


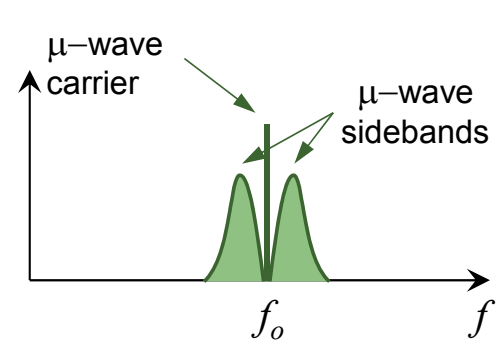

RF domain spectrum

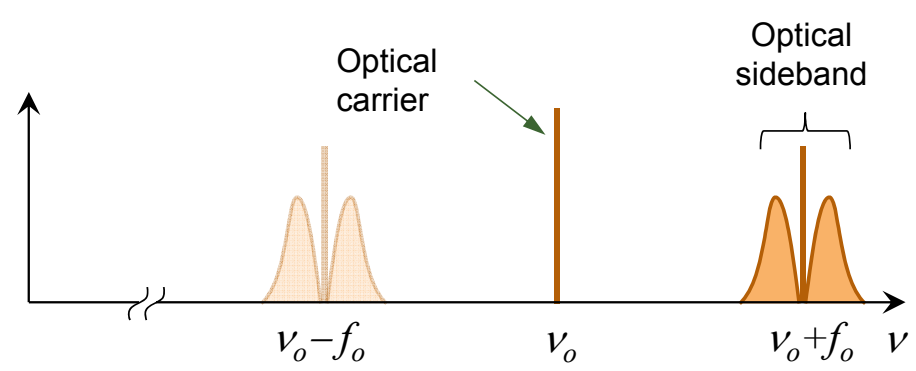

Optical domain spectrum

Figure 2. Spectral contents of the microwave (left) and optical (right) signals, respectively. The entire spectral content of the microwave signal is contained in the optical sidebands, separated from the optical carrier by the microwave carrier frequency. One of the optical sidebands is generally suppressed without any loss of information.

It can be clearly seen in Figure 2 that the microwave modulation only spreads over a fractional part of the microwave carrier frequency, so that the optical sideband is clearly separated from the optical carrier. From this consideration it turns out it may be beneficial to separately perform a transformation on the monochromatic optical carrier and on the optical sideband, using separate functionalities. This way the bandwidth of the function operating on the optical sideband is restricted to the bandwidth of the microwave signal, without the need to cover the full frequency interval between the optical carrier and the optical sideband, which is to a wide extent empty of any spectral information. This approach is particularly effective when using slow light systems, since their delay-bandwidth is essentially restricted to 1 and a larger delay can be obtained if the bandwidth is limited to the strictly needed frequency interval.

The functional separation of the transformations on the optical carrier and its sideband brings on another decisive advantage to turn independent of the value of the microwave carrier frequency, since each optical function is spectrally positioned independently on the carrier and on the sideband. As a result the performance is nominally identical for any microwave carrier frequency.

Slow light systems are basically designated to realize delaying functions ${ }^{2-3}$. An elementary delaying is depicted in Figure 3 for a single frequency signal. The amount of delay can be quantified either by the time delay $\Delta T$ or the phase delay $\Delta \phi$, these 2 quantities being related for a monochromatic wave by the straightforward relation $\Delta \phi=2 \pi f_{o} \Delta T$, where $f_{o}$ is the signal frequency. However, in the situation of a polychromatic signal, as resulting from a broadband modulation, the equivalence no longer holds and the delaying function corresponding to $\Delta \phi=$ constant for all frequencies is designated as phase shifter and that corresponding to $\Delta T=$ constant for all frequencies is called true time delays. These 2 cases are depicted in Figure 3 and it can be seen that true time delays require a linear dependence of the phase shift on the frequency.
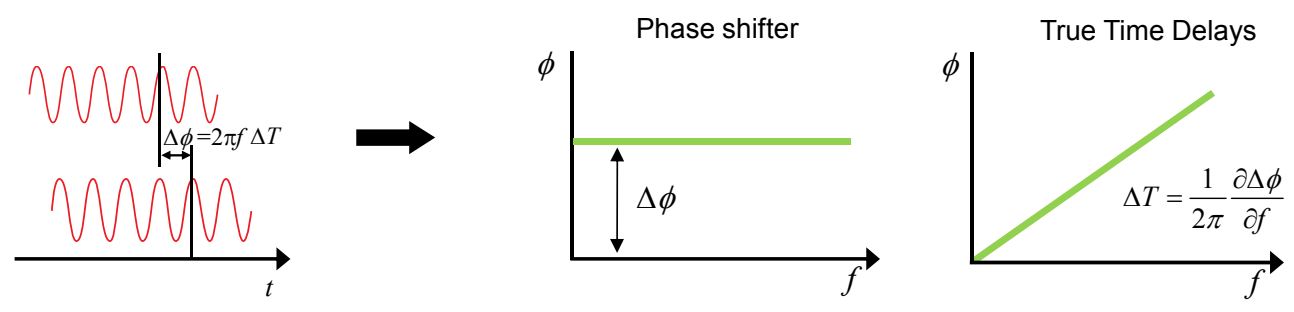

Figure 3. Left: For a single frequency signal phase shift $\Delta \phi$ and time delay $\Delta T$ are closely and unambiguously related. Right: In the case of a signal containing many frequencies, phase shifting and time delaying do not have the same meaning, leading to different functions and resulting to a different dependence of the phase shift on the frequency.

These 2 delaying functions give specific distinct responses in broadband systems, as illustrated in a further section in the case of the notch filter. In particular, only true time delays can actually secure that all frequencies components of the microwave signal are aimed at the same preset direction in phased array antennas, so that the beam remains best collimated by spreading over a narrow angular range. 
Slow light systems based on stimulated Brillouin scattering are very helpful to realize these 2 basic functionalities in microwave photonics, since the delaying is directly proportional to the pump power - giving a linear response that is always a searched property in an analog system - and is directly achieved in the optical fiber conveying the signal. The phase shifting results from the phase response of the Brillouin gain that varies linearly around the peak gain frequency, as shown in Figure 4. In the forthcoming sections it will be explained and illustrated how this feature is the basic tool to realize the phase shifting and true time delaying functions.

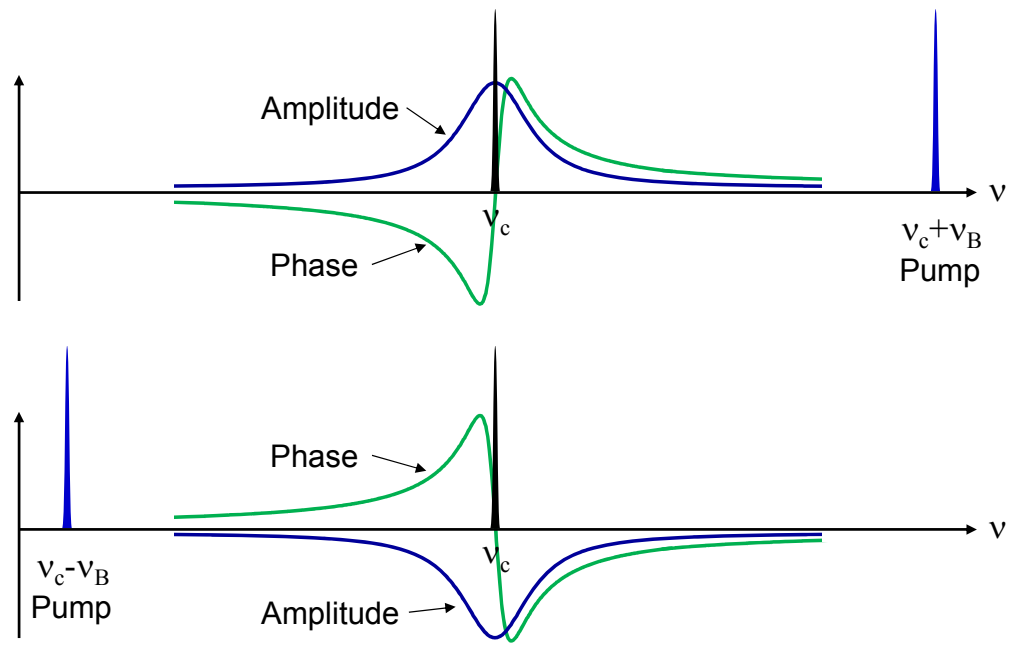

Figure 4. Spectral distribution of the amplitude and phase changes generated by stimulated Brillouin scattering. An optical wave will experience amplification if the medium is pumped by an optical wave at a frequency $v_{\mathrm{B}}$ over the signal frequency $v_{\mathrm{c}}$ (top graph). The pump must propagate in the direction opposite to the signal. If the pump is spectrally placed at a frequency $v_{\mathrm{B}}$ below the signal frequency $v_{\mathrm{c}}$, the signal will be attenuated (bottom graph). The phase shift is zero at the center of the resonance, but follows a linear relationship for small frequency detuning.

\section{PHASE SHIFTER}

A simple and efficient phase shifting function based on stimulated Brillouin scattering was proposed by Loayssa $e t a l^{4}$ in 2006. By examining the amplitude and phase responses depicted in Figure 4, it can be seen that if 2 pumps of identical power are placed at the same spectral distance below and above the signal, the amplitude gain and loss they create will exactly compensate for any detuning of their central resonance frequency. However, their phase change will sum up to double the phase shifting effect. This principle is depicted in Figure 5.

A phase shift can then be applied to any monochromatic wave and be fully optically controlled. This control can be performed by varying the pump power for a fixed detuning $\delta v$ with respect to the center of the Brillouin resonance. It can also be realized by varying the detuning $\delta v$ for a fixed pump power. This latter possibility is more often used in practice. The 2 symmetric pumps are conveniently generated by modulating the monochromatic signal $v_{\mathrm{c}}$ at a frequency $\nu_{\mathrm{B}}+\delta v$ using a wideband electro-optical modulator in a suppressed carrier configuration, so that the 2 modulation sidebands play the role of the 2 pumps, positioned in the spectrum to precisely realize the phase shifting function.

In presence of a microwave photonics signal, as shown in Figure 2 (right), the narrow band property of the Brillouin gain ( $\sim 30 \mathrm{MHz}$ FWHM) makes possible to selectively apply the phase shift to the optical carrier only, so that a global phase shift will be experienced by the beat note between the optical carrier and the optical sideband containing the full microwave signal. This phase shift will be identical for all frequency components of the microwave signal and the exact phase shifting function depicted in Figure 3 is realized. Since the phase shifter selectively acts on the optical carrier only, the phase shift is totally independent of the value of the microwave carrier frequency and no limitation is observed in the microwave carrier frequency and its modulation bandwidth.

A schematic diagram of a practical implementation is shown in Figure 6. All required optical signals and pumps are generated from the same laser source, securing a totally stable spectral positioning of all optical waves, essential for an efficient interaction. A dispersion shifted fiber is used to avoid any biasing phase shifts due to dispersion. 

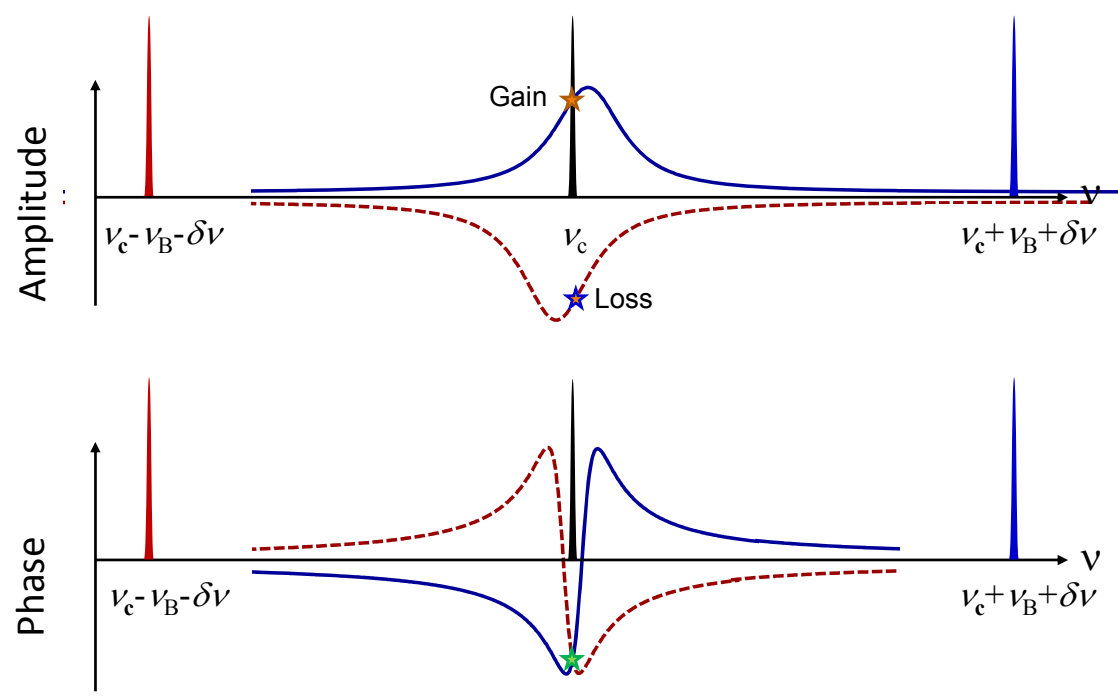

Figure 5. Principle of the phase shifting function realized using stimulated Brillouin scattering in optical fibers. Two optical monochromatic pumps are symmetrically placed at a frequency close to the Brillouin resonance frequency $v_{\mathrm{B}}$ below and above the signal frequency, respectively. If the 2 pumps are slightly but equally detuned by $\delta v$ with respect to the peak central resonance frequency, the generated gain and loss exactly compensate while the phase changes sum up.

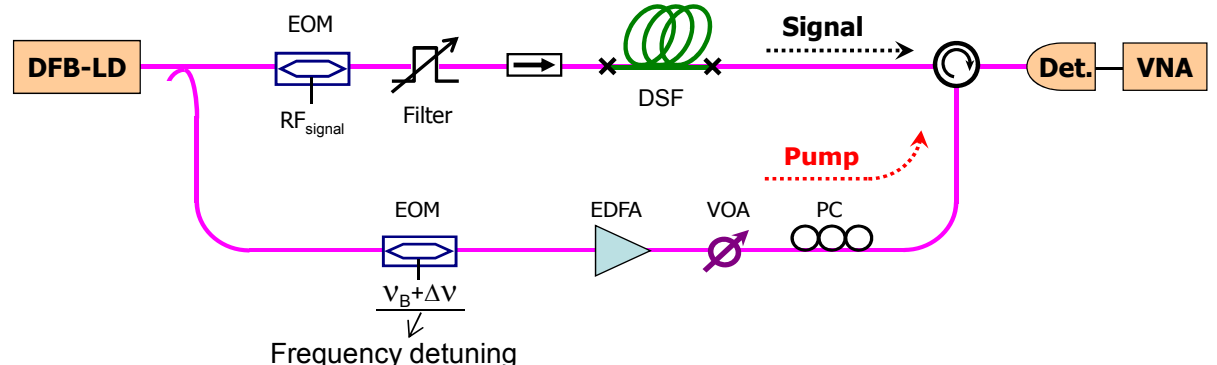

Figure 6. Schematic diagram of a microwave phase shifter realized using the principle depicted in Figure 5 in an optical fiber. The 2 pumps for the Brillouin interaction are generated from the same laser source modulated by the microwave signal, in a distinct fiber channel using an electro-optic modulator. EOM: electro-optic modulator, EDFA: erbium doped fiber amplifier, VOA: variable optical attenuator, DSF: dispersion-shifted fiber, VNA: vector network analyzer.
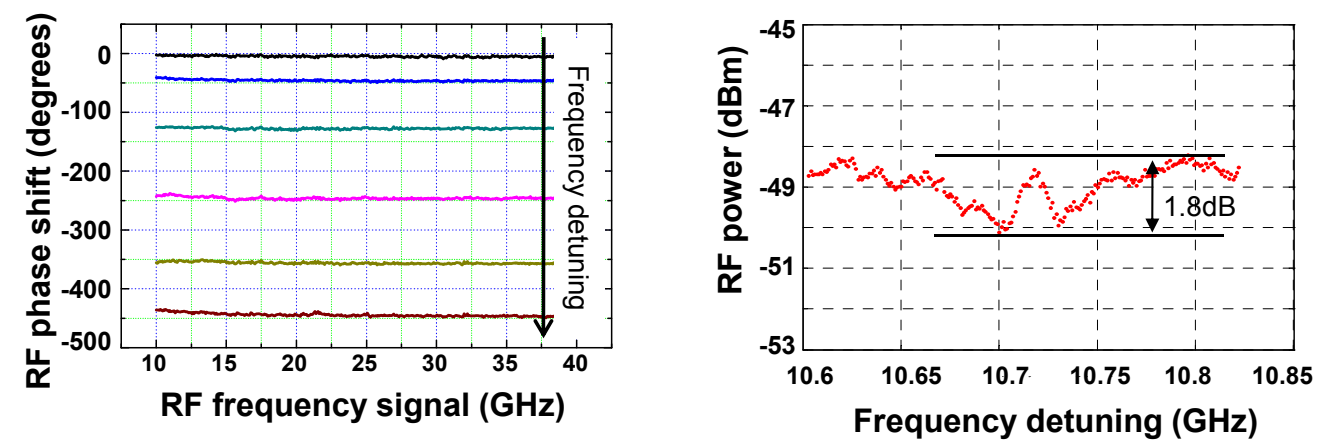

Figure 7. Left: Phase shifts obtained for different microwave carrier frequency between 10 and $40 \mathrm{GHz}$, showing the total absence of dependence on the frequency. The phase shifts cover a range exceeding $400^{\circ}$. Right: amplitude change of the microwave signal for different frequency detuning, corresponding to the phase shift range shown on the left graph. 
The phase shifts obtained using this simple phase shifter are shown in Figure 7, for a wide range of microwave carrier frequency. The total absence of dependence on the frequency is clearly demonstrated over a wide frequency range covering 2 octaves, simply limited by the instrumentation. A continuous phase shift range larger than $400^{\circ}$ is achieved, far exceeding the minimum required coverage of a full signal period.

This phase shifter can be considered as a function by itself, but it is also an essential building block of the efficient true time delaying function described in the next section.

\section{TRUE TIME DELAYS}

The true time delay function is realized when all frequency components experience a phase shift proportional to their frequency spacing with respect to the carrier, as depicted in Figure 3 (right). To properly realize this function in microwave photonics, this linear relation must be valid for all signal components in the optical domain, including the optical carrier. This means that the slow light interaction giving this linear phase dependence on frequency must extend over a spectral range scaled by the microwave carrier frequency, as shown in Figure 8 (left). But it is well known that the phase shift slope - so the delaying power - scales in an inverse proportion to the interaction bandwidth, limiting the delay-bandwidth product to something close to unity.

Actually, since the microwave modulation bandwidth is in general a small fraction of the microwave carrier frequency, a wide extent of the spectral range covered by the interaction contains no signal spectral component and the interaction bandwidth is inefficiently exploited. A smart approach ${ }^{5-6}$ consists in only covering the optical sideband with the slow light generating interaction - so over a bandwidth given by the microwave modulation bandwidth, much smaller than the microwave carrier frequency - and by adjusting the phase of the optical carrier to extrapolate the linear phase shift applied over the entire optical sideband, as shown in Figure 8 (right). This approach is designated as the separate carrier tuning technique.

This way the true time delaying is just applied over the strictly required bandwidth and no delaying power is wasted over spectral regions containing no spectral information. The phase tuning on the carrier is normally performed using the phase shifter described in the previous section.

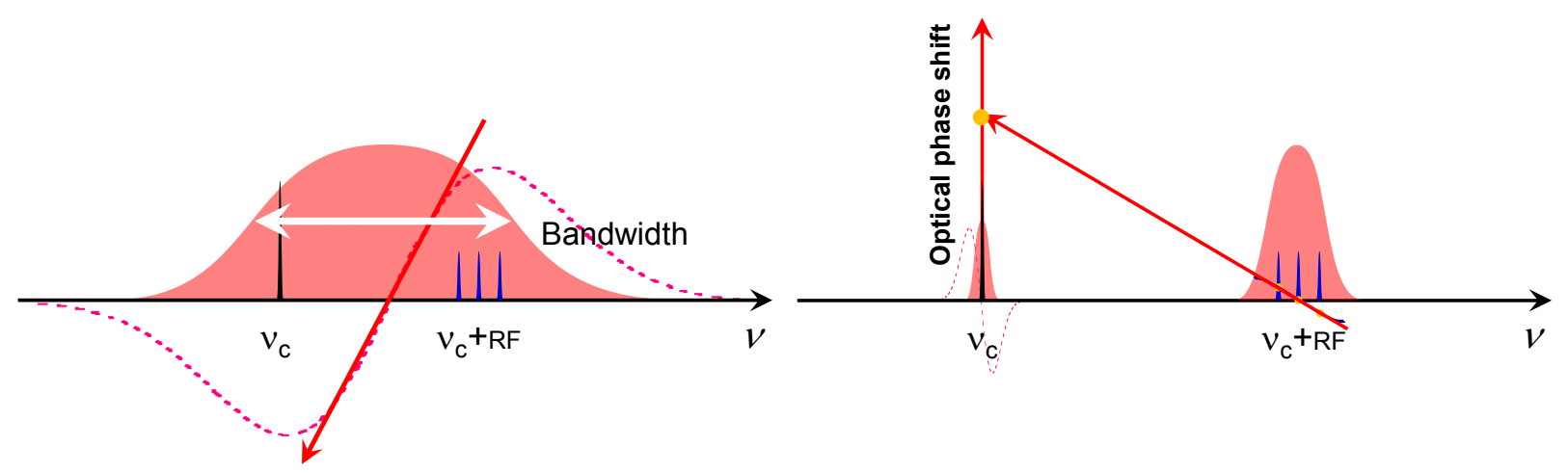

Figure 8. Left: Direct true time delaying using a slow light interaction covering the full bandwidth occupied by the optical signal. A wide spectral range covered by the interaction contains no spectral information.

Right: True time delaying realized using the separate carrier tuning technique. The interaction covers only the optical sideband and the phase of the carrier is separately adjusted to match the linear frequency dependence of the phase shifts.

The practical realization of this separate carrier tuning technique requires the implementation of two functions in the same system: a true time delaying over the optical sideband and a separate pure phase shifting over the carrier. As a demonstration this has been implemented following the experimental layout shown in Figure 9, where the true time delays are realized using a Brillouin slow light system and the phase shifting is performed using the principle described in the previous section. Using this system true time delays up to $10 \mathrm{~ns}$ were achieved for a $6 \mathrm{GHz}$ microwave signal modulated over a $120 \mathrm{MHz}$ bandwidth ${ }^{6-7}$. Here again the separate carrier tuning technique makes possible to obtain true time delays with a magnitude independent of the value of the microwave carrier frequency. 


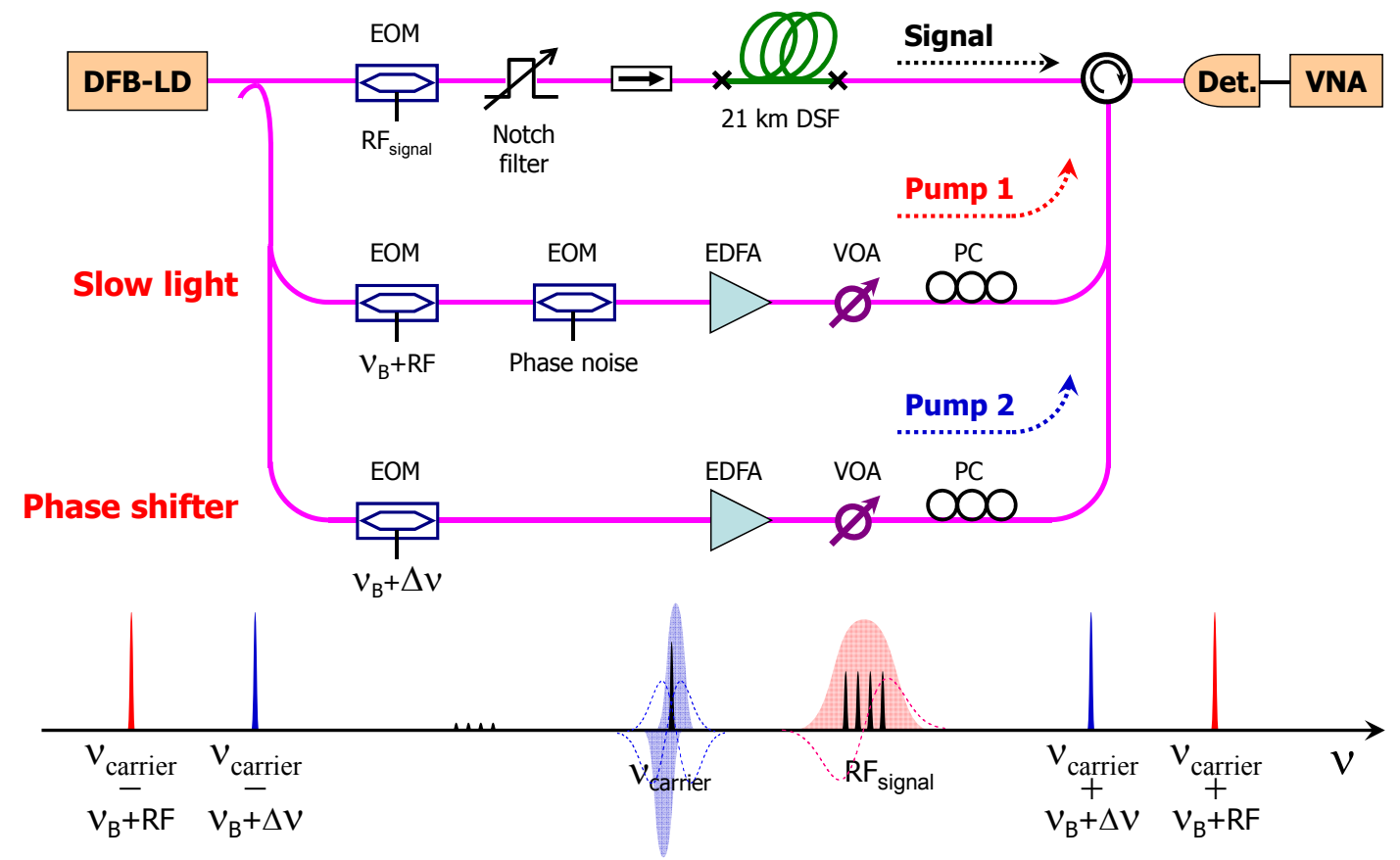

Figure 9. Top: Diagram of the experimental setup realizing true time delays using the separate carrier tuning technique. The true time delaying on the optical sideband is realized using a Brillouin slow light system with a spectrally broadened pump $^{7}$ to cover the bandwidth of the microwave modulation. The phase shifting is implemented using the system described in Section 2. All optical signals (pumps and optical carrier) are generated from the same laser source.

Bottom: Graphical representation of all optical signals present in the system, with the 3 combined Brillouin interactions.

\section{APPLICATION: NOTCH FILTER}

As an illustration the true time delay system described in the previous section has been implemented in the simple filter configuration $^{8}$ depicted in Figure 10. The transfer function of such a filter shows periodic notches with a frequency spacing given by the delay between the two arms of the incoherent interferometer. This spacing can thus be adjusted by applying a true time delay between the arms. The absolute frequency position of the notches is determined by the phase difference between the arms for the microwave signal. This position can therefore be tuned by applying a phase shifting for the microwave signal in one of the arms.

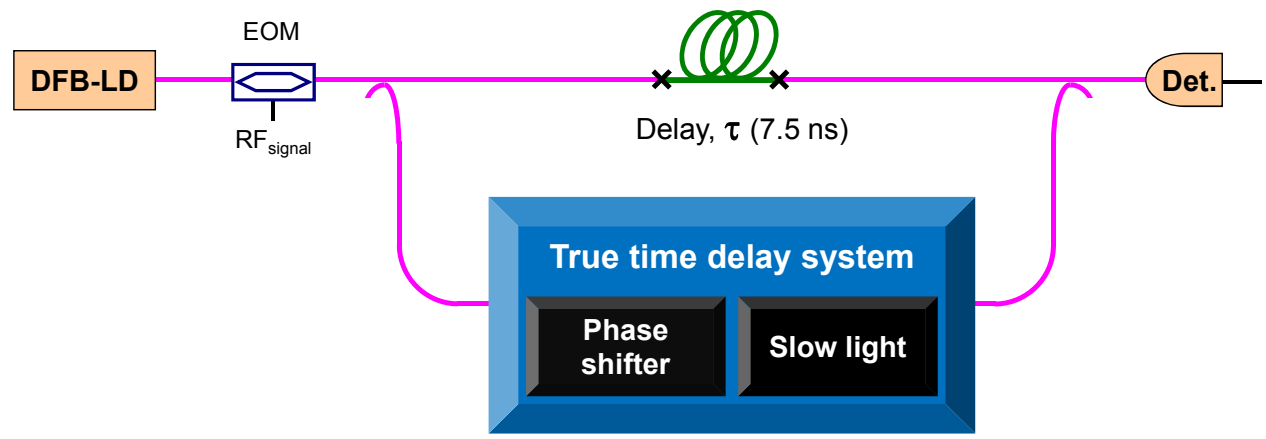

Figure 10. Schematic diagram of a dynamic microwave photonics notch filter. The frequency position of the notches can be tuned with the phase shifter, while the frequency spacing between the notches can be modified by applying a true time delaying in one of the arms. 
A maximum true time delay of $10 \mathrm{~ns}$ at $6 \mathrm{GHz}$ over a $120 \mathrm{MHz}$ bandwidth is obtained using a pump power of $110 \mathrm{~mW}^{6}$. This corresponds to a $20 \%$ change of the frequency spacing between the notches (from $26.7 \mathrm{MHz}$ to $22.5 \mathrm{MHz}$ ) requiring a carrier phase compensation of $302.4^{\circ}$. The measured spectral transfer function of this dynamic filter is shown in Figure 11.
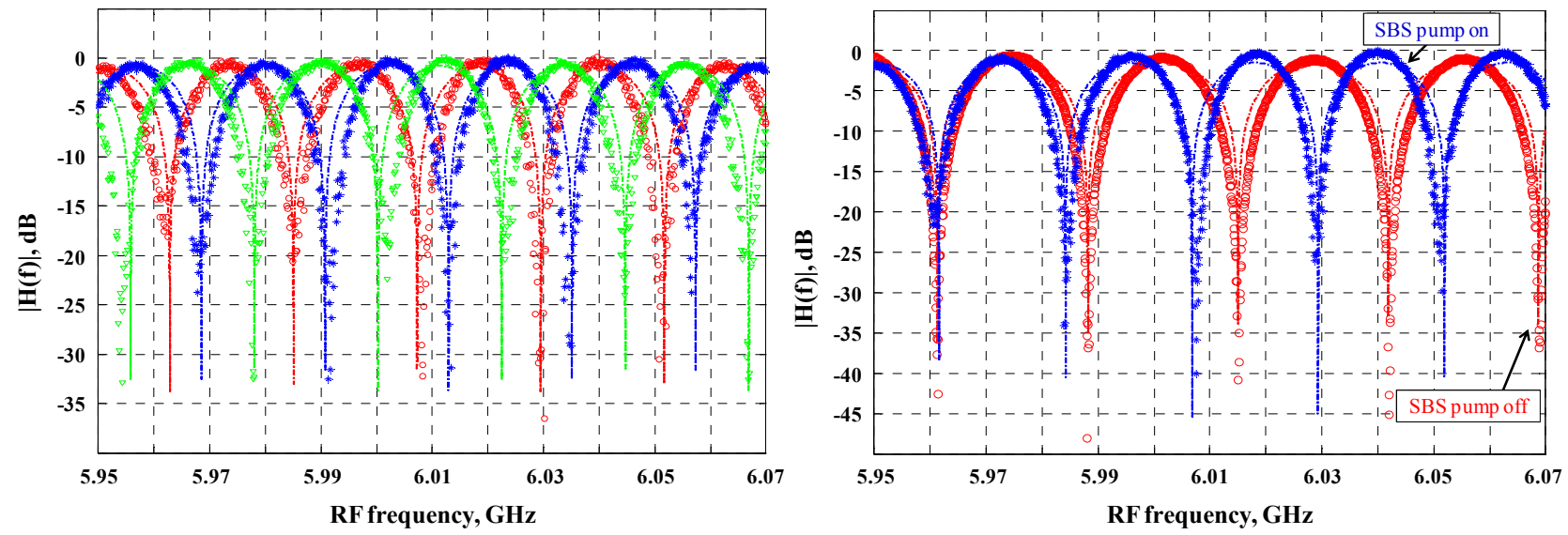

Figure 11. Left: Notch filter response for 3 different phase shifts applied to the optical carrier, showing the possibility to dynamically position a notch at any frequency thanks to the phase shifting capability over more than $360^{\circ}$.

Right: Notch filter response with and without true time delaying, demonstrating the possibility to substantially adjust the spectral periodicity of the notch response (20\%).

\section{CONCLUSION}

We have presented a simple experimental conceptual demonstration that Brillouin slow light elements offer novel and outstanding capabilities in microwave photonics systems, particularly thanks to the innovative all-optical tunable truetime delay line based on the separate carrier tuning technique. We have implemented two stimulated Brillouin scattering systems to separately manage the dispersion characteristics at the optical carrier and RF subcarrier frequencies. A continuously tunable true time delay from 0 to $10 \mathrm{~ns}$ has been achieved, with a $120 \mathrm{MHz}$ instantaneous bandwidth, and at an operating frequency of $6 \mathrm{GHz}$. It must be stressed that this technique has no physical limitation on the operating RF frequency since the Brillouin resonance can be positioned at any desired RF frequency.

\section{ACKNOLEDGEMENTS}

We acknowledge the support from the Swiss National Science Foundation through project 200020-121860 and from the European Community's Seventh Framework Program [FP7/2007-2013] under grant agreement n 219299 (GOSPEL project).

\section{REFERENCES}

[1] J. Capmany and D. Novak, "Microwave photonics combines two worlds," Nat Photon 1(6), 319-330 (2007)

[2] R. W. Boyd and D. J. Gauthier, "'Slow" and "fast" light," in Progress in Optics, pp. 497-530 (2002)

[3] L. Thevenaz, "Slow and fast light in optical fibres," Nat Photon 2(8), 474-481 (2008)

[4] A. Loaysa and F. J. Lahoz, "Broad-band RF photonic phase shifter based on stimulated Brillouin scattering and single-sideband modulation," Photon. Technol. Lett., 18, 208-210 (2006).

[5] P. A. Morton and J. B. Khurgin, "Microwave Photonic Delay Line With Separate Tuning of the Optical Carrier," Photon. Technol. Lett., 21, 1686-1688 (2009). 
[6] S. Chin, L. Thévenaz, J. Sancho, S. Sales, J. Capmany, P. Berger, J. Bourderionnet and D. Dolfi, "Broadband true time delay for microwave signal processing, using slow light based on stimulated Brillouin scattering in optical fibers," Opt. Express 18(21), 22599-22613 (2010)

[7] M. González Herráez, K. Y. Song and L. Thévenaz, "Arbitrary-bandwidth Brillouin slow light in optical fibers," Opt. Express 14, 1395-1400 (2006)

[8] J. Sancho, C. Sanghoon, M. Sagues, A. Loayssa, J. Lloret, I. Gasulla, S. Sales, L. Thevenaz and J. Capmany, "Dynamic Microwave Photonic Filter Using Separate Carrier Tuning Based on Stimulated Brillouin Scattering in Fibers," Photonics Technology Letters, 22, 1753-1755 (2010) 